Equilibrium fluctuations of liquid state static properties in a subvolume by molecular dynamics

D. M. Heyes', D. Dini' , and E. R. Smith'

Citation: The Journal of Chemical Physics 145, 104504 (2016); doi: 10.1063/1.4962165

View online: http://dx.doi.org/10.1063/1.4962165

View Table of Contents: http://aip.scitation.org/toc/jcp/145/10

Published by the American Institute of Physics

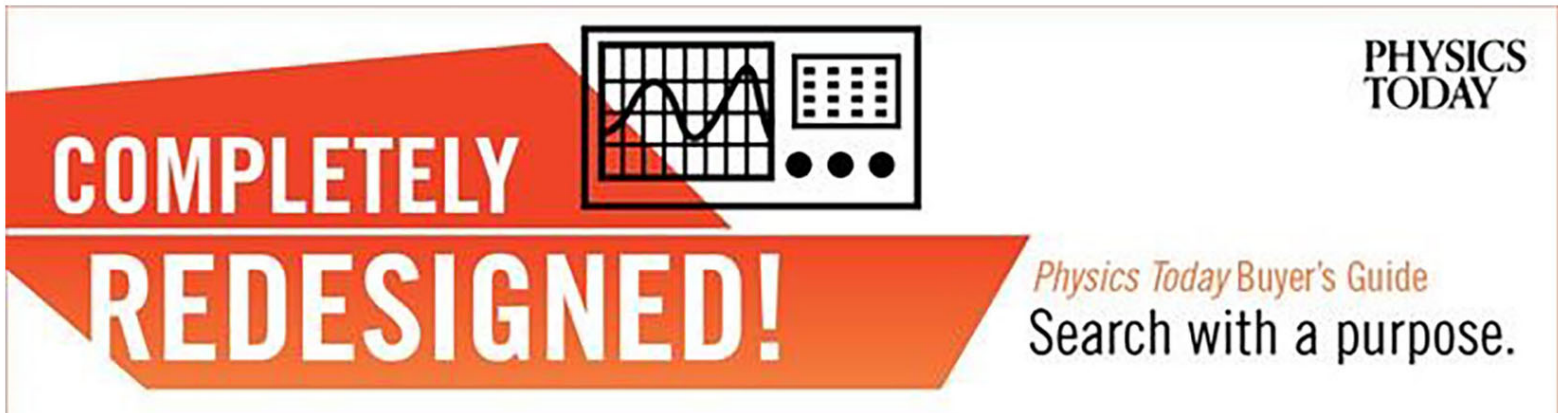




\title{
Equilibrium fluctuations of liquid state static properties in a subvolume by molecular dynamics
}

\author{
D. M. Heyes, ${ }^{1, a)}$ D. Dini, ${ }^{1, b)}$ and E. R. Smith ${ }^{2, c)}$ \\ ${ }^{1}$ Department of Mechanical Engineering, Imperial College London, Exhibition Road, South Kensington, \\ London SW7 2AZ, United Kingdom \\ ${ }^{2}$ Department of Chemical Engineering, Imperial College London, Exhibition Road, South Kensington, \\ London SW7 2AZ, United Kingdom
}

(Received 11 May 2016; accepted 22 August 2016; published online 13 September 2016)

\begin{abstract}
System property fluctuations increasingly dominate a physical process as the sampling volume decreases. The purpose of this work is to explore how the fluctuation statistics of various thermodynamic properties depend on the sampling volume, using molecular dynamics (MD) simulations. First an examination of various expressions for calculating the bulk pressure of a bulk liquid is made, which includes a decomposition of the virial expression into two terms, one of which is the Method of Planes (MOP) applied to the faces of the cubic simulation cell. Then an analysis is made of the fluctuations of local density, temperature, pressure, and shear stress as a function of sampling volume (SV). Cubic and spherical shaped SVs were used within a spatially homogeneous LJ liquid at a state point along the melting curve. It is shown that the MD-generated probability distribution functions (PDFs) of all of these properties are to a good approximation Gaussian even for SV containing only a few molecules $(\sim 10)$, with the variances being inversely proportional to the SV volume, $\Omega$. For small subvolumes the shear stress PDF fits better to a Gaussian than the pressure PDF. A new stochastic sampling technique to implement the volume averaging definition of the pressure tensor is presented, which is employed for cubic, spherical, thin cubic, and spherical shell SV. This method is more efficient for less symmetric SV shapes. Published by AIP Publishing. [http://dx.doi.org/10.1063/1.4962165]
\end{abstract}

\section{INTRODUCTION}

Molecular Dynamics (MD) simulation has been a standard tool of condensed matter physics since its invention in the 1950s. ${ }^{1}$ One of the main growth areas of particlebased computer simulations of liquids since the 1970s has been the development of coarse-grained techniques such as Brownian Dynamics (BD), Stokesian Dynamics (SD), and Dissipative Particle Dynamics (DPD) for dispersed phase systems. ${ }^{2}$ The characteristic units of distance and time used in these techniques are much larger than those associated with the individual molecule's diameter and dynamical time scale. There has been interest in developing coarse graining techniques which operate at the boundary between $\mathrm{MD}$ and these more continuum descriptions. For example, in molecular-continuum coupling, a region of the physical system which can be simulated by Molecular Dynamics (MD) is positioned next to a region represented by Computational Fluid Dynamics (CFD) with the same coupling cell size for the two fluid descriptions. ${ }^{3-5}$ An overlap region is used to exchange information between the CFD and MD regions, which provides a smooth transition zone between the two.

The properties of the molecular system need to be averaged over time to give a sufficiently accurate value to

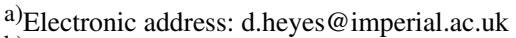

b)Electronic address: d.dini@imperial.ac.uk

c)Electronic address: edward.smith05@imperial.ac.uk
}

pass on to the continuum description. The computer time required for the averaging will depend on the size of the MD cell. It is therefore of interest to explore how the fluctuations in various key molecular derived quantities such as density, kinetic energy (temperature), and pressure tensor depend on the sampling volume (SV), which is the main theme of this work. The required information to be passed could be based on their probability distribution functions (PDFs) and their lower moments. Fluctuating hydrodynamics has been used to introduce stress fluctuations in the continuum representation. ${ }^{5-7}$ The focus here is to explore the fluctuation statistics on the molecular side of the boundary. The SV acts as an open system, and is not in a well defined thermodynamic ensemble even though the simulation cell as a whole may be subject to imposed constraints to generate the properties of a particular ensemble. How the SV property fluctuation statistics depend on the simulation ensemble used is also explored here.

Fluctuations in static (i.e., thermodynamic and mechanical) properties have been studied by MD for many years, but almost always taking the sampling volume to be the whole MD or Monte Carlo (MC) simulation cell. For example, the interaction parts of all thermodynamic properties can be defined in terms of the probability function of the potential energy. ${ }^{8,9}$ The shear stress fluctuations of block averages of the whole system can yield the shear viscosity. ${ }^{10-13}$ System property fluctuations are fundamental to our understanding of nonequilibrium states, ${ }^{14,15}$ although in this study equilibrium liquids are considered exclusively. 
As the sampling volume or scale of scrutiny decreases, system property fluctuations increasingly dominate physical processes. The purpose of this work is to explore the extent to which the fluctuation statistics of various thermodynamic properties, which are described for bulk systems in terms of statistical mechanical formulas, apply to small sampling volumes just a few molecules across. The average values are independent of sampling volume size, but as will be shown, some departures from the bulk formulas for the fluctuations are observed when they are applied to small finite volumes, especially for the local pressure. Nevertheless, it is shown that for all the properties and definitions, even for sampling volumes containing $\sim 10$ molecules, Gaussian statistics still hold reasonably well. The variances can be specified in terms of effective thermomechanical constants which take account of the "granularity" of the liquid. Trends in the molecular-level fluctuations are presented which could be useful in developing liquid system theories and computational methods valid on these length scales.

In Section II pressure tensor definitions based on the virial formula and derived for the entire domain are compared numerically. Various local pressure tensor definitions are also reviewed and explored. A new method for computing the pressure by the Volume Averaging (VA) method is presented and used, which involves a stochastic selection process to obtain the fraction of the length of the line between two molecules which falls within the SV. In Section III results are presented for the PDF and its lower moments for the molecule number density, temperature, pressure, and shear stress defined in a subvolume of the whole domain. Conclusions are made in Sec. IV.

\section{THEORY}

In this section various methods for calculating the local pressure tensor are presented and compared. Consider a cubic simulation cell of sidelength, $s$, volume, $V$, and containing $N$ molecules, which is subject to periodic boundary conditions (PBCs) along the three Cartesian directions. A homogeneous single component monatomic fluid is considered in which the molecules interact through a radially symmetric pair potential, $\phi(r)$, where $r$ is the separation between the centers of the two molecules.

The relevant literature can be considered to start from the Irving and Kirkwood (IK) equation for the pressure tensor at point, $\underline{r}^{16}$

$$
\begin{aligned}
\underline{\underline{P}}^{I K}(\underline{r})= & \sum_{i=1}^{N} \frac{1}{m} \underline{p}_{i} \underline{\underline{p}} \delta \delta\left(\underline{r}-\underline{r}_{i}\right)+\frac{1}{2} \sum_{i=1}^{N} \sum_{j \neq i}^{N} \underline{r}_{i j} \underline{f}_{i j} \\
& \times \int_{0}^{1} \delta\left(\underline{r}-\underline{r}_{i}+\lambda \underline{r}_{i j}\right) d \lambda,
\end{aligned}
$$

where the integral in the last term follows a straight line contour between the two molecules, and $\delta$ is the Dirac delta function. The momentum of molecule, $i$, of mass $m$, is $\underline{p}_{i}$. The pair separation vector is $\underline{r}_{i j}=\underline{r}_{i}-\underline{r}_{j}$, where $\underline{r}_{i}$ and $\underline{r}_{j}$ are the coordinates of the two molecules. The pair force between the molecules is $\underline{f}_{i j}=-\left(\underline{r}_{i j} / r_{i j}\right) d \phi\left(r_{i j}\right) / d r_{i j}$.
The kinetic contribution to the local pressure tensor is the first term on the right hand side of Eq. (1), which can be reduced for equilibrium systems to $\underline{\underline{P}}_{k}=\rho k_{B} T \underline{\underline{I}}$, where $k_{B}$ is Boltzmann's constant, $\rho=N / V$, and $\underline{\underline{I}}$ is the unit tensor. This formula involves an arbitrary interaction path between two molecules, ${ }^{17,18}$ which means that in IK, and those methods based on it, the spatially local pressure tensor is not uniquely defined. ${ }^{19,20}$

The integral of Eq. (1) over a subvolume leads to the Volume Average (VA) local pressure tensor definition, ${ }^{21}$

$$
\begin{aligned}
\underline{\underline{P} \Omega} & =\int_{\Omega} \underline{\underline{P}}^{I K} d V \\
& =\sum_{i=1}^{N} \frac{1}{m} \underline{p}_{i} \underline{p}_{i} \vartheta_{i}+\frac{1}{2} \sum_{i=1}^{N} \sum_{j \neq i}^{N} \underline{r}_{i j} \underline{f}_{i j} \int_{0}^{1} \vartheta_{\lambda} d \lambda \\
& =\sum_{i=1}^{N} \frac{1}{m} \underline{p}_{i} \underline{p}_{i} \vartheta_{i}+\frac{1}{2} \sum_{i=1}^{N} \sum_{j \neq i}^{N} \underline{r}_{i j} f_{i j} l_{i j},
\end{aligned}
$$

where if $\Omega$ is the volume of the SV domain, $\vartheta_{i}$ $=\int_{\Omega} \delta\left(\underline{r}-\underline{r}_{i}\right) d V, \quad \vartheta_{\lambda}=\int_{\Omega} \delta\left(\underline{r}-\underline{r}_{i}+\lambda \underline{r}_{i j}\right) d V, \quad$ and $l_{i j}$ $=\int_{0}^{1} \vartheta_{\lambda} d \lambda$. The whole space can be divided into a mesh of such subvolumes and the microscopic pressure tensor evaluated as an average within each of these. The configurational part of the pressure tensor is the focus of this work, as the kinetic part is a straightforward extension of the virial route.

The widely used virial formula (VIR), ${ }^{22-28}$ for bulk system molecular simulations can be recovered from Eq. (2) by making the subvolume the entire domain (i.e., the periodically repeated cell),

$$
\underline{\underline{P}} V=\sum_{i=1}^{N} \frac{1}{m} \underline{p}_{i} \underline{p}_{i}+\frac{1}{2} \sum_{i=1}^{N} \sum_{j \neq i}^{N} \underline{r}_{i j} \underline{f}_{i j},
$$

which could therefore be considered to be a special case of VA. The configurational part of the pressure tensor, $\underline{\underline{P}}_{c}$, the second term on the right hand side of Eq. (3), can be written as

$$
\underline{\underline{P}}_{c}=\frac{1}{2 V} \sum_{i=1}^{N} \sum_{j \neq 1}^{N}\left(\underline{r}_{0, i j}-\underline{s}_{i j}\right) \underline{f}_{i j},
$$

where $\underline{r}_{0, i j}=\underline{r}_{0, i}-\underline{r}_{0, j}$ and $\underline{r}_{0, i}$ and $\underline{r}_{0, j}$ are the coordinates of molecules $i$ and $j$ in the origin simulation cell. $\underline{s}_{i j}$ is the PBC pair separation displacement. For each Cartesian direction, $\alpha$, $s_{\alpha, i j}= \pm s$ or 0 , which enforces the nearest image convention to ensure that $r_{i j} \leq s / 2$ (we assume that there are no long range Coulomb interactions here).

Note that the pair force term denoted by $\underline{f}_{i j}$ is always calculated using the nearest image convention applied to the pair separations. The two terms in Eq. (4) separated are

$$
\underline{\underline{P}}_{c}^{i}=\frac{1}{2 V} \sum_{i=1}^{N} \sum_{j \neq i}^{N} \underline{r}_{0, i j} \underline{f}_{i j},
$$

which we call the internal virial, IV, tensor and,

$$
\underline{\underline{P}}_{c}^{e}=-\frac{1}{2 V} \sum_{i=1}^{N} \sum_{j \neq i}^{N} \underline{s}_{i j} \underline{f}_{i j},
$$


which we call the external virial (EV). The EV expression can be considered to be a form of the Method of Planes (MOP) definition, ${ }^{29}$ (see below) in which the pair forces cross the six faces of the simulation cell. The EV term is the contribution to $\underline{\underline{P}}_{c}$ from those pair interactions that cross the (virtual) boundaries of the central or origin simulation cell.

The IV expression of Eq. (5) for the whole domain can be simplified further,

$$
2 V \underline{P}_{c}^{i}=\sum_{i=1}^{N} \sum_{j=1}^{N} \underline{r}_{0, i j} \underline{f}_{i j}=2 \sum_{i=1}^{N} \underline{r}_{0, i} \underline{F}_{i},
$$

where the total force on molecule $i$ is $\underline{F}_{i}=\sum_{j \neq i} f_{i j}$, and $f_{i i}=0$ (see the supplementary material for further details of the intermediate steps). Because the positions and forces are bounded in magnitude, $\left\langle\underline{\underline{P}}_{c}^{i}\right\rangle$ (where $\langle\cdots\rangle$ represents a time or ensemble average) will be zero after sufficient system averaging on grounds of symmetry.

As originally derived, ${ }^{29}$ the MOP method involves computing the forces across an infinite plane, for the interaction part of the pressure tensor. A series of such planes spaced parallel to one of the simulation cell faces (surface normal, say, in the $x$-direction) is used here, and referred to simply as the "MOP" construction. For the $x \alpha$ element of the configurational part of the pressure tensor, $P_{x \alpha}$ is

$P_{x \alpha}\left(X_{k}\right)=\frac{1}{2 A} \sum_{i=1}^{N} \sum_{j \neq i}^{N} f_{\alpha i j}\left(\operatorname{sgn}\left(x_{i}-X_{k}\right)-\operatorname{sgn}\left(X_{k}-x_{j}\right)\right)$,

where $\alpha$ is any of the Cartesian directions, $A=s^{2}$ is the cross-sectional area in the $y z$ plane, $X_{k}$ is the location of the $k$ th plane and $\operatorname{sgn}(x)=1$ for $x>0, \operatorname{sgn}(x)=0$ for $x=0$, and $\operatorname{sgn}(x)=-1$ for $x<0$. The contributions from the $i j$ interactions which extend into a neighboring periodic image cell need to be included using the usual periodic boundary "wrap around" construction. MOP gives the tractions across each plane; for example, for a plane whose perpendicular is in the $x$ direction, only the elements $P_{x x}, P_{x y}$, and $P_{x z}$ can be obtained from the MOP procedure. When summed over the faces of a cube in the EV adaptation, the full $3 \times 3$ pressure tensor is assembled.

The equivalence between MOP and VA for infinitely thin slabs of infinite extent in the two other Cartesian directions in the plane has been proved.$^{30}$ The relative merits of using the virial method and MOP for bulk systems have been discussed by Heinz et al. ${ }^{31,32}$ By inserting a row of virtual planes parallel to one of the faces of the simulation cell, the formal equivalence between the VIR and MOP routes to the bulk pressure tensor has also been proved. ${ }^{31}$

The Lennard-Jones (LJ) potential used in this study is

$$
\phi_{L J}(r)=4 \epsilon\left[(\sigma / r)^{12}-(\sigma / r)^{6}\right],
$$

where $\epsilon$ and $\sigma$ are the characteristic energy of interaction and diameter of the molecule, respectively. For the LJ potential the long range correction (LRC) to the pressure of an equilibrium spatially homogeneous system calculated from the virial formula for the bulk fluid is $P_{L R C}=\left(32 \pi \rho^{2} / 9\right) \epsilon\left[\left(\sigma / r_{c}\right)^{12}\right.$ $\left.-(3 / 2)\left(\sigma / r_{c}\right)^{6}\right]$, where $r_{c}$ is the interaction truncation distance (see page 67 in Ref. 33). The treatment of the long range correction for the MOP halfspace geometry is given in supplementary material.

The VA method requires $l_{i j}$ in Eq. (2) to be evaluated for a given SV shape. This is analytic for a spherical SV, ${ }^{34}$ and can be achieved exactly for a cubic SV with ray tracing over the six surface planes of the cube. ${ }^{35}$ An alternative approach proposed here is to choose a uniformly distributed random number, $\lambda_{R}$, in the range $0 \leq \lambda_{R} \leq 1$, and the new formula, $l_{i j}=\int_{\Omega} \delta\left(\underline{r}-\underline{r}_{i}+\lambda_{R} \underline{r}_{i j}\right) d V$ follows. The weighting factor, $l_{i j}$, is either unity or zero depending on whether the random point on the line between molecules $i$ and $j$ (i.e., at the position, $\underline{r}^{\prime}=\underline{r}_{i}-\lambda_{R} \underline{r}_{i j}$ ) is within the SV or not (respectively). Only if $\underline{r}$ is contained within the SV does that $i j$ interaction for the current assembly configuration contribute to the VA pressure accumulator. This "stochastic" variant implementation of VA averaged over a simulation is statistically equivalent to the formal evaluation of $l_{i j}$ obtained by trigonometry for each $i j$ pair at each time step. The stochastic route to $l_{i j}$ is competitive with the trigonometric approach in determining all geometric crossings, as the latter requires additional code in the two particle forces calculation, which is computationally demanding apart from in the sphere $\mathrm{SV}$ case.

The size of the VA subvolume, $\Omega$, is $4 \pi R^{3} / 3$ for the sphere and $8 R^{3}$ for the cube. The new implementation of VA can also be applied to shell shaped SV, where, for example, the SV is the zone between two concentric spheres of radius, $\delta_{R} R$, and $R$, where $0 \leq \delta_{R}<1$, and for two cubes with coincident centers and orientations with sidelengths, $2 \delta_{R} R$ and $2 R$. The $\delta_{R} \rightarrow 0$ limit of the shell VA implementation is the original VA case defined in Eq. (2), while the other limit, $\delta_{R} \rightarrow 1^{-}$ is the very thin spherical or cubic shell subvolume solution. The shell version of the VA definition of the local pressure is referred to as "VASH." The value $\delta_{R}=0.99$ was used for computational sampling efficiency. The term "VA" is reserved for the volume averaging method of Eq. (2) where $\Omega$ is the entire cube or sphere.

For a thin spherical shell subvolume of $\simeq 4 \pi R^{2} \Delta R$, with $\Delta R=\left(1-\delta_{R}\right) R$, the thickness of the shell, and the mean radius of the shell, is $\simeq R(>>\Delta R)$. Let $R$ be the vector from the center of the sphere to the surface. If $P_{N}$ is the normal and, $P_{T}$, the tangential component, ${ }^{36-40}$ of the pressure tensor then,

$$
\underline{\underline{P}}(\underline{R})=P_{N}\left[\underline{\hat{e}}_{R} \underline{\hat{e}}_{R}\right]+P_{T}\left[\underline{\hat{e}}_{\theta} \underline{\hat{e}}_{\theta}+\underline{\hat{e}}_{\phi} \underline{\hat{e}}_{\phi}\right],
$$

where $\underline{\hat{e}}_{R}, \underline{\hat{e}}_{\theta}$, and $\underline{\hat{e}}_{\phi}$ are the orthogonal vectors of the spherical coordinate system. Also,

$$
\begin{aligned}
& P_{N}=\left(\frac{1}{8 \pi R^{2} \Delta R}\right) \sum_{i=1}^{N} \sum_{j \neq i}^{N} r_{i j} \cos ^{2}(\theta) f_{i j} l_{i j}^{\prime}, \\
& P_{T}=\left(\frac{1}{16 \pi R^{2} \Delta R}\right) \sum_{i=1}^{N} \sum_{j \neq i}^{N} r_{i j} \sin ^{2}(\theta) f_{i j} l_{i j}^{\prime},
\end{aligned}
$$

where $l_{i j}^{\prime}=1$ if the randomly chosen vector, $\underline{r}_{i j}^{\prime}$, falls within the shell, and 0 otherwise. The extra factor of 2 in the denominator in the two formulas of Eq. (11) is to avoid double counting of the pair interactions. The second factor 
a)

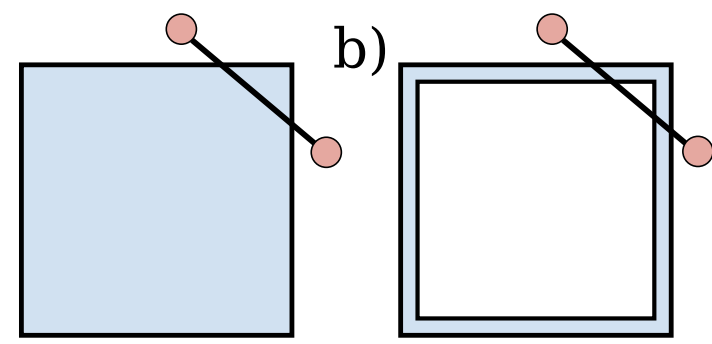

C)
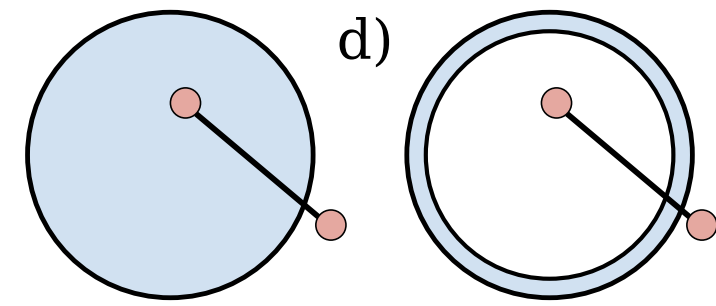

FIG. 1. The various variants of the Volume Averaging (VA) (see Eq. (2)) volumes used in this work, (a) Cubic volume, VA, (b) cubic shell volume, VASH, (c) spherical volume, VA, and (d) spherical shell volume, VASH. The black bars with pink circles at their ends indicate two molecules and the line between their centers. The blue shaded areas are the subvolumes for each case.

of 2 in the denominator in the formula for $P_{T}$ is because there are two mutually orthogonal tangential directions and terms for each $i j$ pair. The trace of the pressure tensor for the VA shell is $\left(P_{N}+2 P_{T}\right) / 3$ in spherical polar coordinates, or $\left(P_{x x}+P_{y y}+P_{z z}\right) / 3$, in Cartesian coordinates. Figure 1 illustrates the VA and VASH methods for a spherical and cubic subvolume.

The atomic virial (AVIR) local pressure tensor definition decomposes the virial form of Eq. (3) into single molecule components within the SV,

$$
\underline{\underline{P}} \Omega=\sum_{i=1}^{N} \frac{1}{m} \underline{p}_{i} \underline{p}_{i} \vartheta_{i}+\frac{1}{2} \sum_{i=1}^{N} \vartheta_{i} \sum_{j \neq i}^{N} \underline{r}_{i j} f_{i j} .
$$

The $\vartheta_{i}$ operator selects those molecules whose centers, $\underline{r}_{i}$, are in the subvolume. AVIR has been widely used for bulk liquids, ${ }^{41}$ and glasses, ${ }^{42}$ even though it appears not to be derivable from the IK formula of Eq. (1) and gives rise to spurious pressure oscillations if there are density oscillations in the system. ${ }^{29,43}$ Nevertheless, it is computationally efficient to implement and involves spatial averaging within the SV as does the VA method of Eq. (2).

A local pressure tensor-like quantity can be defined as an extension of the IV bulk pressure tensor definition of Eq. (7),

$$
\underline{P}_{c}^{i v} \Omega=\frac{1}{2} \sum_{i=1}^{N} \vartheta_{i} \sum_{j \neq i}^{N} \underline{r}_{0, i j} \underline{f}_{i j} .
$$

A molecule $i$ in Eq. (13) only contributes if $\underline{r}_{i}$ is within the subvolume.

\section{RESULTS AND DISCUSSION}

Only the configurational part of the local pressure tensor is considered as the kinetic component in each case follows directly from the kinetic theory of gases. The computed

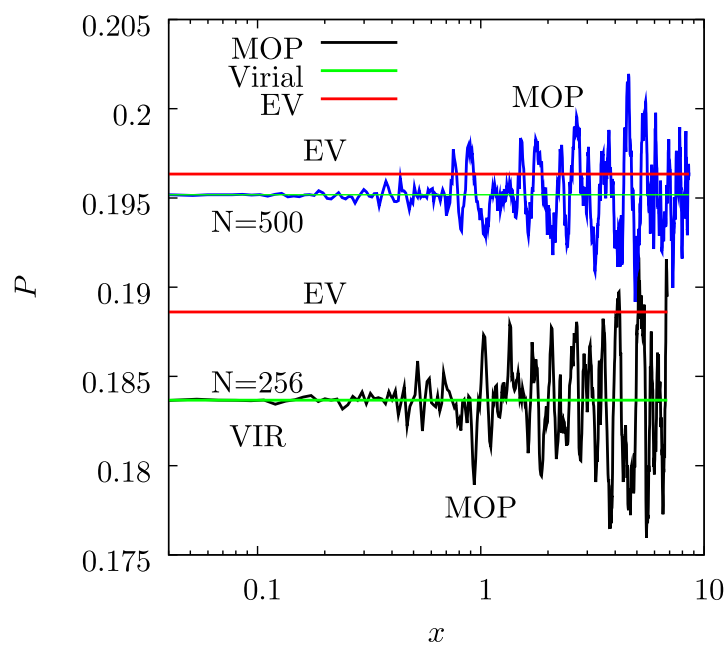

FIG. 2. Accumulated configurational part of the pressure as a function of the distance between the planes, $x$ in the MOP method of Eq. (8). "VIR" is the virial method from Eq. (3), and EV is from Eq. (6). There were $N=256$ and 500 molecules in the simulation cell of LJ molecules, and $\rho=0.8$ and $T=1.0$. The maximum value of $R$ is $s / 2$. The truncation distance was $r_{c}=2.5$ and the pair force used to generate the particle trajectories was tapered to zero in the range $2.5 \leq r \leq 2$. . The long range correction based on $r_{c}=2.5$ of -0.684 was included in the quoted values. The simulation length was $t=100$ for both system sizes. Note the log-lin scale.

quantities are given in $\mathrm{LJ}$ reduced units based on $\sigma, \epsilon, m$. The force truncation distance, $r_{c}$, was $3.5 \sigma$, unless stated. The Verlet leapfrog algorithm was used in the MD code to integrate the equations of motion, ${ }^{33}$ with a time step of 0.005. Simulations were carried out using either velocity rescaling ${ }^{44,45}$ or Nosé-Hoover ${ }^{46-48}$ thermostats applied to all molecules in the system. Some simulations were conducted at constant total energy (NVE). All the state points considered were in the liquid part of the LJ phase diagram. Figures 2 and 3 are for $\rho=0.8$ and $T=1.0$, and the other figures were for $\rho=0.9157$ and $T=1.0$ which is on the solid-fluid phase boundary. ${ }^{49}$ The simulations were carried out with $N=2048$

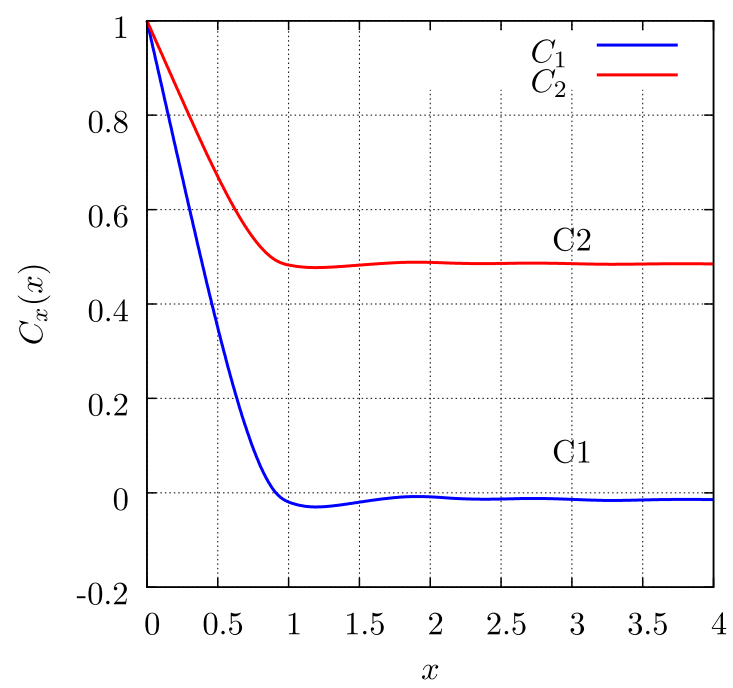

FIG. 3. The correlation functions defined in Eq. (14) of the MOP plane pressure as a function of plane separation, $x$, for a simulation cell of 864 LJ molecules, and $\rho=0.8$ and $T=1.0$. 
and $4000 \mathrm{LJ}$ particles to assess the system size dependence of the calculated quantities.

This section starts by considering the pressure of the entire domain, in Subsection III A. Next, the pressure of subvolumes of variable size is discussed in Section III B. The probability density functions are explored in Subsection III C.

\section{A. The pressure of the entire domain}

In this section the pressure associated with the entire simulation cell calculated from the virial and routes is focused on. Figure 2 shows the average configurational part of the pressure, $P_{c}$, calculated by the MOP formula of Eq. (8) as a function of $x$, the separation distance between adjacent planes. The virial and EV cell averaged values are given as horizontal lines. The fluctuations in the MOP pressure about the virial pressure decrease with reducing $x$, as the number of planes sampled per time step is $s / x$. In the $x$-direction for $N=500$ the mean separation between the molecules is 0.017 in a Cartesian direction parallel to one of the cell sides. Figure 2 shows that in the vicinity of $x=0.017$ the MOP and virial routes are statistically indistinguishable, so there is nothing to be gained statistically by using a finer resolution.

The EV average pressure, Eq. (6), is seen in Fig. 2 to be systematically higher than the virial and MOP values, indicating that the IV term is negative in both cases. The figure shows that the virial expression for the pressure from Eq. (3) and MOP converges much faster to the correct value. The difference between the virial and $\mathrm{EV}$ values is smaller for $N=500$ than $N=256$. Both simulations were for 100 reduced time units. Although the IV term from Eq. (7) is zero after sufficient statistical averaging, this can take an impractically long time, which is especially significant for state points where the configurational part of the pressure is small in magnitude. Small cell periodic systems are known to exhibit long equilibration times. ${ }^{50-55}$ The relative importance of the IV component (see Eq. (5)) of the total virial decreases with increasing density as the mean pressure increases. For example, for the melting line state point the absolute value of this internal pressure term is $\leq 0.003$ for $500 \leq N \leq 1024$, while the average virial pressure from Eq. (3) is $2.865 \pm 0.001$. Also for the purely repulsive Weeks-Chandler-Andersen (WCA) potential, ${ }^{56}$ a simulation at the same density and temperature, over a similar simulation time, gave an internal pressure absolute error which was less than 0.001 while the average pressure was 5.79 , so EV and the virial formulas give good agreement in that case. Although the virial method is probably the most statistically efficient and reliable pressure measurement, the EV route could also be computationally advantageous because it only requires those interactions that cross the cell boundaries, provided the system sizes and mean configurational pressure are not too small.

The degree to which the configurational part of the instantaneous pressure value at a plane is correlated with its value on neighboring planes is quantified by the following two spatial pressure correlation functions, $C_{1}(x)$ and $C_{2}(x)$ :

$$
\begin{aligned}
C_{1}(x) & =\frac{\langle(P(0)-\bar{P})(P(x)-\bar{P})\rangle}{\left\langle(P(0)-\bar{P})^{2}\right\rangle}, \\
C_{2}(x) & =\frac{\langle P(0) P(x)\rangle}{\left\langle P(0)^{2}\right\rangle},
\end{aligned}
$$

where $C_{1}$ involves the difference in the instantaneous value of the MOP pressure, Eq. (8), for a given plane from the mean pressure, $\bar{P}$. This function uses the fluctuation about the mean. In contrast, the function $C_{2}$ uses the absolute MOP pressure value for each plane. Let $x^{\prime}$ be the coordinate along the $x$-direction. These functions represent the spatial autocorrelation function of the instantaneous pressure at a plane at $x^{\prime}=0$ at time $t$ multiplied by its value from the plane at $x^{\prime}=x$ further away at the same time. The two functions are calculated at each time step, $k$, and then are averaged over the simulation of $n_{t}$ time steps (i.e., $C_{1}(x)=\sum_{k=1}^{n_{t}} C_{1}(x, k) / n_{t}$, for time step $k$, where $1 \leq k \leq n_{t}$ ).

Figure 3 shows that $C_{1}(x, t)$ decays monotonically and initially almost linearly with increasing $x$ until it is statistically almost zero for ca. $x \geq 2$. It is slightly negative for this system size in the large $x$ limit presumably because a large (say) positive pressure fluctuation about the mean in one region of the system associated with a higher than average local density must be compensated for by a negative pressure fluctuation about the mean in the rest of the system where the density is lower than the average.

\section{B. Mean pressure in a subvolume smaller than the entire domain}

The local pressure associated with a subvolume of magnitude less than or equal to that of the whole domain (i.e., simulation cell) is considered in this section. Figure 4 shows the average value of the configurational part of the pressure, $P_{c}$, as a function of $R$ calculated by the atomic virial (AVIR) (see Eq. (12)) and internal pressure (see Eq. (13)) virial based routes. To minimize correlations between successive time steps the molecules in the cell were uniformly shifted by variable extents and the periodic boundary conditions applied, to ensure the center of the subvolume enclosed substantially different configurations at each time step. The radial distribution function is shown in the figure to help gauge the lengthscales. Figure 4 shows that AVIR datasets give the virial pressure from all $R$ values down from $R=s / 2$, whereas the subvolume extension of this quantity, $\mathrm{IV}(\mathrm{R})$, defined in Eq. (13), only agrees with the bulk virial value up to ca. $R=5$. In the $R \rightarrow s / 2$ limit the local internal pressure formula for the cube has a small maximum before decreasing rapidly towards the internal pressure value of the whole cell (which is very close to zero on the scale of the figure), as expected in that limit. There is also a small maximum close to $R \rightarrow s / 2$ for the spherical $\mathrm{IV}(\mathrm{R})$ case of Eq. (13), but which still appears to terminate close to the bulk virial value at $R=s / 2$. In the $R=s / 2$ cube case the subvolume fills the entire cell volume, whereas for the sphere with $R=s / 2$, the subvolume is only an inscribed sphere, and there is in effect a buffer region of molecules not in the subvolume close to the cell boundary which mitigates to a certain extent the effect of the PBC. Figure 5 shows the average pressure for cubic 


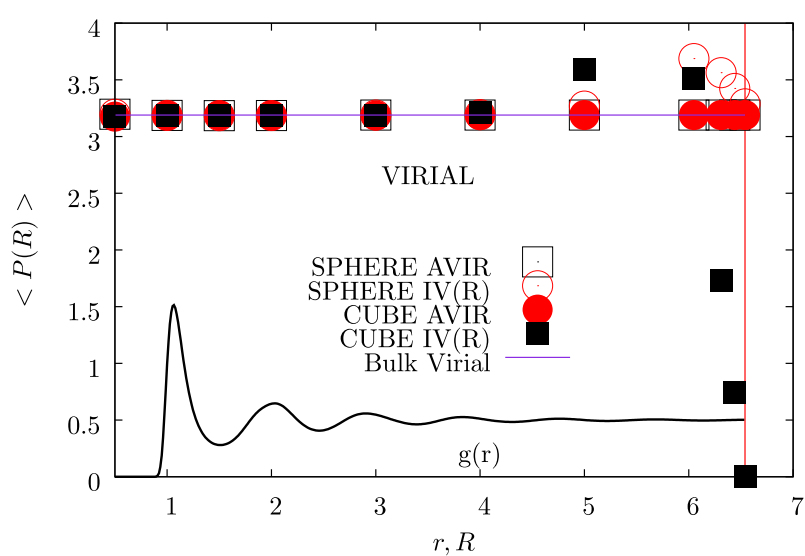

FIG. 4. The average configurational part of the pressure as a function of $R$ for cubic and spherical subvolumes given according to the virial based local pressure tensor definitions. The state point is $\rho=0.9157$ and $T=1.0,{ }^{49}$ with $N=2048$. The interaction truncation distance is $s / 2=6.5$. In each case the configurational part of the pressure is shown without including the long range correction to the pressure $\left(P_{L R C}=-0.328\right)$. Key: The annotation, "SPHERE AVIR" is the atomic virial trace of the pressure tensor for the spherical subvolume using the definition of the pressure tensor given in Eq. (12), and "CUBE AVIR" denotes the same quantity for a cubic subvolume. The annotation "SPHERE IV(R)" is the internal virial component of the cubic subvolume from Eq. (13), and "CUBE IV(R)" is the same quantity for the cubic subvolume. The virial pressure including the long range correction is 2.865. "Bulk Virial" is the virial pressure of the whole system using the formula in Eq. (3).

and spherical subvolume geometries for the VA of Eq. (2) and VASH form, showing that there are no anomalous features for $R$ close to $s / 2$. Note that those interactions that cross the boundary of the periodic cell pass through the subvolume twice, and have to be counted two times in the averaging process.

\section{Probability distribution functions for subvolumes}

The mean pressure shown in Figs. 4 and 5 is the first moment of a (normalized) probability distribution function (PDF) of pressure values, $W\left(P_{c}\right)$. In the thermodynamic limit

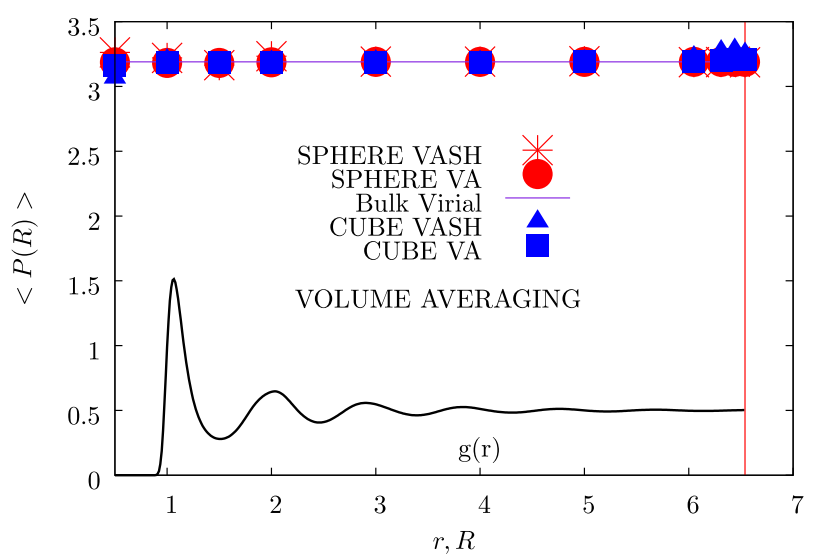

FIG. 5. As for Fig. 4 except that local pressure definitions based on the volume averaging (VA) approach are considered. Key: "VASH" is the trace of the pressure tensor from the shell VA definition, with $\delta_{R}=0.99$; "VA" is the trace of the volume averaging method of Eq. (2); "Bulk Virial" is the virial pressure of the whole system using the formula in Eq. (3). The radial distribution function, $g(r)$, is also shown. the PDF of a property, $X$, is given by the Gaussian or normal distribution form ${ }^{57}$

$$
W(X)=\frac{1}{\left(2 \pi \sigma_{2}\right)^{1 / 2}} \exp \left(-(\delta X)^{2} / 2 \sigma_{2}\right),
$$

where $\delta X=X-\bar{X}$ and $\bar{X} \equiv \sigma_{1}$ is the average value of $X$, according to the central limit theorem limit. The first moment of the distribution is $\bar{X}$ and $\sigma_{2}$ is the second moment or variance of the distribution (the standard deviation is $\left.\left(\sigma_{2}\right)^{1 / 2}\right)$. The PDF of the dimensionless quantity, $X^{\prime}=X / \bar{X}$, is $W\left(X^{\prime}\right)=\bar{X} W(X)$. For example, for the local mean density $\rho / \bar{\rho}$, where $\rho$ is the average number density in the subvolume, $\Omega$, and $\bar{\rho}=N / V$. The dimensionless temperature distribution is $T / \bar{T}$, where $T=\sum_{i=1}^{M} m_{i} \underline{v}_{i}^{2} / 3 k_{B}$ is the average temperature of the molecules in the subvolume. The reduced pressure, $X^{\prime} \equiv P / \bar{P}$, probability distribution is also considered.

The variance of each property in Eq. (15) is conveniently expressed in terms of the thermodynamic limit formula. For example, if the average number of molecules in subvolume $\Omega$ is $M$, then for the reduced number density,

$$
\sigma_{2}(\rho / \bar{\rho})=\sigma_{2}(\Omega / \bar{\Omega})=\left(\frac{1}{M}\right)\left(\frac{\beta_{T}}{\beta_{T, 0}}\right)=\frac{Q_{\rho}}{M},
$$

taken from Ref. 9, p. 54, Eq. (2.88), Ref. 58, p. 60 Eqs. (3.23) and (3.24), and also Refs. 59 and 60. The variance, $\sigma_{2}(\Omega / \bar{\Omega})$, is for the distribution of $\Omega$ containing exactly $M$ molecules. The isothermal compressibility of the liquid is $\beta_{T}=(\partial \rho / \partial P)_{T} / \rho$, and $\beta_{T, 0}=P^{-1}$ is the ideal gas form. The isothermal thermodynamic bulk compressional modulus $K_{T}$ is $\beta_{T}^{-1}$. A "softness" parameter, $Q_{\rho}=\beta_{T} / \beta_{T, 0}$, is defined in Eq. (16) which has a maximum value of 1 for an ideal gas. Equation (16) substituted in Eq. (15) reveals that the reduced density PDF becomes narrower with an increasing number of molecules in the SV, i.e., the liquid becomes less compressible on that scale as the system becomes more coarse grained.

For the temperature, (see, for example, Ref. 58, p. 62 Eq. (3.27), Refs. 61 and 62),

$$
\sigma_{2}(T / \bar{T})=\left(\frac{2}{3 M}\right)\left(\frac{c_{0, v}}{c_{v}}\right)=\frac{2 Q_{T}}{3 M},
$$

where $c_{v}$ is the heat capacity at constant volume and $c_{0, v}=1.5$ is its ideal monatomic gas value, $Q_{T}=c_{0, v} / c_{v}$, and for an open system, ${ }^{63} Q_{T}=1$. The normalized temperature PDF becomes narrower with increasing SV. For the pressure, ${ }^{64}$ after some rearrangement,

$$
\begin{aligned}
\sigma_{2}(P / \bar{P}) & =\left(\frac{2}{3 M}\right)\left(\frac{P_{0}}{\bar{P}}\right)^{2}\left(\frac{K_{\infty}-K_{0}}{K_{\infty, 0}-K_{0,0}}\right) \\
& =\left(\frac{2}{3 M Q_{P}}\right)\left(\frac{P_{0}}{\bar{P}}\right)^{2},
\end{aligned}
$$

where $P_{0}$ is the pressure of the ideal gas, $K_{\infty}$ is the infinite frequency compressional modulus, and $K_{0}$ is the zero frequency adiabatic compressional modulus. ${ }^{65}$ The quantities, $K_{\infty, 0}$ and $K_{0,0}$ are those for the ideal gas, where $K_{\infty, 0}-K_{0,0}=2 \rho k_{B} T / 3$. The softness parameter in this case is $Q_{P}=\left(K_{\infty, 0}-K_{0,0}\right) /\left(K_{\infty}-K_{0}\right)$. Equation (18) indicates that the normalized local pressure PDF becomes narrower as the number of molecules in the subvolume increases. The variance 
increases with $K_{\infty}-K_{0}$, and is infinite for inverse power fluids in the hard sphere limit. ${ }^{66}$

The Gaussian distribution form of the PDF will eventually break down for very small SV because the more general skewed Poisson statistics will apply, and excluded volume effects will become increasingly important. The local number density PDF where the subvolume, $\Omega$, can be a cube, sphere, or a shell with the same shape but with a thickness $\left(1-\delta_{R}\right) R$. In the $\delta_{R} \rightarrow 1^{-}$limit the volume of the (thin) shell is $\Omega_{s h}=3\left(1-\delta_{R}\right) \Omega$, where $\Omega$ is the volume of the cube or sphere. The normalized local density PDFs for the full geometry volume and its thin shell counterpart on the outer boundary are in the large system limit,

$W\left(\frac{\rho}{\bar{\rho}}, \Omega\right)=\left(\frac{\bar{\rho} \Omega}{2 \pi Q_{\rho}}\right)^{1 / 2} \exp \left(-\left[\frac{\rho}{\bar{\rho}}-1\right]^{2} \bar{\rho} \Omega / 2 Q_{\rho}\right)$

and

$$
\begin{aligned}
W\left(\frac{\rho}{\bar{\rho}}, \Omega_{s h}\right)= & \left(\frac{\bar{\rho} \Omega}{2 \pi Q_{\rho}}\right)^{1 / 2}\left[\left(3\left(1-\delta_{R}\right)\right]^{1 / 2}\right. \\
& \times \exp \left(-3\left[\frac{\rho}{\bar{\rho}}-1\right]^{2} \bar{\rho} \Omega\left[1-\delta_{R}\right] / 2 Q_{\rho}\right),
\end{aligned}
$$

respectively. The parameter $Q_{\rho}$ can be set to 1 , being the ideal gas approximation, which is the upper limit, giving the broadest PDF whereas $Q_{\rho}=\rho k_{B} T \beta_{T}<1$ for the actual simulated liquid. As the subvolume decreases in size the normalized PDF becomes more spread out and (hence) because of the normalization, shallower and tends towards the baseline for all $\rho / \bar{\rho}$. This trend is accentuated for the shell or $\Omega_{s h}$ case.

Figure 6 shows the MD-generated PDFs of the normalized local density for the cube with $R=1.5$ and 4 , which are compared with the prediction of the Gaussian formulas of Eq. (19) for the cube. The PDFs of Eq. (20) for the cube thin shell case (not shown) are much broader. The cubic subvolume contains on average 25 and 469 molecules for

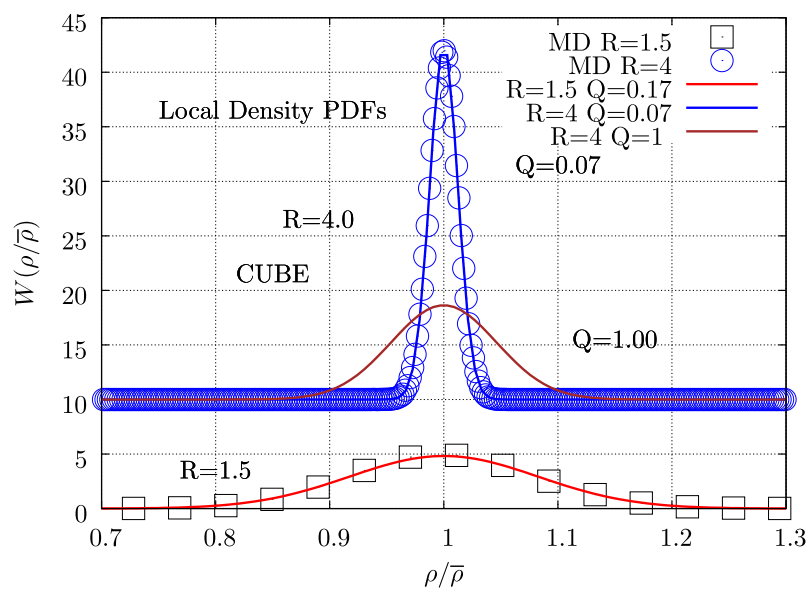

FIG. 6. The normalized local density probability distribution function, $W(\rho / \bar{\rho})$. The simulation is for a cubic subvolume for $R=1.5$ (lower frame) and 4.0 (upper shifted frame). The state point is $\rho=0.9157$ and $T=1.0$. There were $N=2048$ molecules in the simulation cell and $r_{c}=3.5$. A Nosé-Hoover thermostat with a time constant of 3 was used. The MD cube $\mathrm{PDF}$ (annotated, "MD") is compared with Eq. (19) using $Q_{\rho}=0.07$ (where $\left.\beta_{T}=0.077\right)$ and the ideal gas limit, $Q_{\rho}=1$.

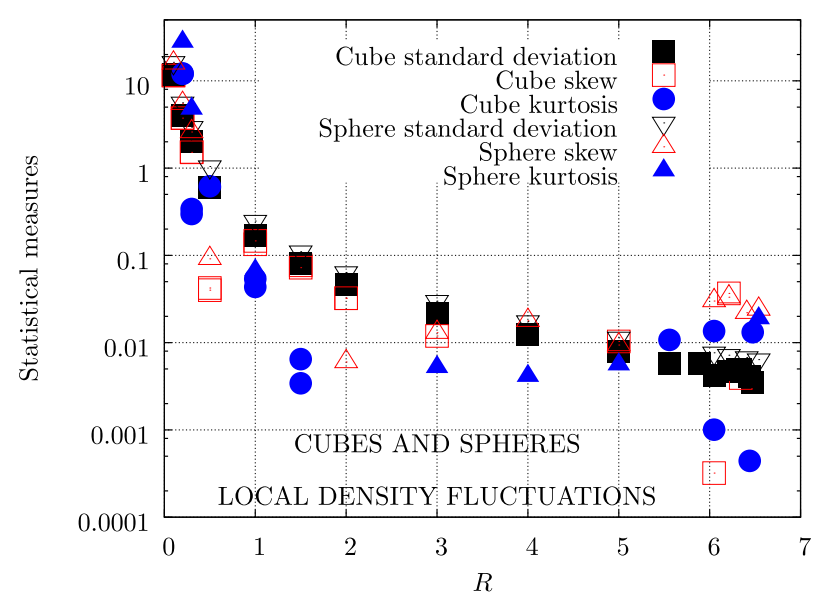

FIG. 7. The standard deviation, $\sigma_{2}^{1 / 2}$, skew, and kurtosis of the probability distribution function of the local density, $\rho / \bar{\rho}$ of the subvolume as a function of $R$. Data for cubic and spherical subvolumes are shown. The state point is $\rho=0.9157$ and $T=1.0$ for $N=2048$. The volume of the cube is $8 R^{3}$ and the (inscribed) sphere is $4 \pi R^{3} / 3$. Note the lin-log scale.

$R=1.5$ and 4 , respectively. The Gaussian functional form given in Eq. (20) matches the MD data well. The Kolafa and Nezbeda LJ equation of state, ${ }^{67}$ of the Benedict-WebbRubin (MBWR) analytic form, gives $\beta_{T}=0.0314$, which is equivalent to an isothermal bulk modulus of 31.8 , and therefore $Q_{\rho}=\rho k_{B} T \beta_{T}=0.0288$. This proves to be too "stiff" in Eq. (19) to reproduce the $R=4 \mathrm{MD} \mathrm{PDF}$, and good agreement is obtained using a smaller compressional modulus, of 13.0, which corresponds to $Q_{P}=0.070$, as may be seen in Fig. 6. The figure also shows that the ideal gas limit, $\beta_{T}=0.916$, gives a PDF which is much broader than the MD generated function. In the MD case for $R=4$ the large occupancy effects restrict the extent of density fluctuation within the given volume, so the density distribution is narrower. For $R=1.5$ the best fit $Q_{P}=0.17$ or the bulk modulus is 5.4 which is therefore tending to the ideal gas limit. Too much physical significance should not be given to the "effective" thermodynamic functions derived from the observed fluctuations in the limit $\Omega \rightarrow 0$, as their use is to represent the effects of small SV and excluded volume interactions between the molecules.

The shape of a PDF can be described by the second to fourth moments, $\sigma_{2}, \sigma_{3}$, and $\sigma_{4}$, respectively. The breadth, degree of slant to the right, and "peakiness" of the PDF, respectively, increase when the variance, $\sigma_{2}$, skew, $\sigma_{3} / \sigma_{2}^{3 / 2}$, and kurtosis, $\sigma_{4} / \sigma_{2}^{2}-3$ become more positive. ${ }^{57}$ Figure 7 shows these statistical measures for the PDF of the local density plotted on a lin-log scale. They all decrease in magnitude with increasing subvolume. For ca. $R>1$, the skew and kurtosis are statistically very small, $\sim 0.01$, indicating a near Gaussian PDF. Although the statistics deteriorate for $R$ smaller than 1 , it is clear that all three statistical measures become more positive as $R \rightarrow 0$, indicating a PDF more peaked than Gaussian, with a long tail for $\rho / \bar{\rho}>1$ (skew).

Figure 8 gives the variance $\sigma_{2}(\rho / \bar{\rho})$ as a function of $\Omega^{-1}$. The three lines show $k_{B} T \beta_{T} \Omega^{-1}$ for $\beta_{T}^{-1}=0.916$ (ideal gas limit), 13.0, and 31.8 (the bulk liquid value). The MD data 


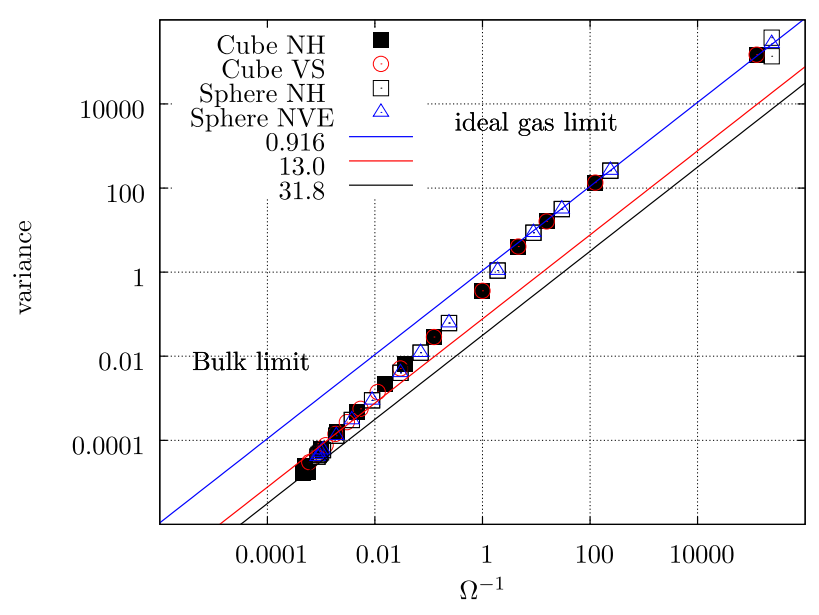

FIG. 8. The variance $\sigma_{2}(\rho / \bar{\rho})$ as a function of $\Omega^{-1}$ for cubic and spherical subvolumes. The MD data are taken from simulations carried out under constant temperature by Velocity Rescaling ("VS") and Nosé-Hoover (NH) methods, and constant total energy (NVE) constraints. The three lines show $T \beta_{T} \Omega^{-1}$ for $\beta_{T}^{-1}=0.916$ (ideal gas), 13.0, and 31.8 (the bulk value). The state point details are the same as for Fig. 7. Note the log-log scale.

are from simulations carried out with constant temperature using the velocity rescaling and Nosé-Hoover thermostats, and constant total energy (NVE) dynamics. In the low $\Omega$ limit the value of the ("effective") compressibility which fits the simulation data for cubes and spheres approaches the ideal gas value, and tends to the bulk liquid value as $\Omega$ approaches the volume of the simulation cell. When $R=s / 2$ the variance drops sharply to zero for a cubic subvolume, as the number of molecules in the simulation cell is exactly $N$ in that limit.

Figure 9 presents the local temperature probability distribution function, $W(T / \bar{T})$, for a cubic subvolume with $R=1$ and 4 . The symbols are for NVE MD data, and the continuous curve is the Gaussian analytic form taking the ideal gas heat capacity, i.e., the variance in Eq. (17) has $Q_{T}=1$.

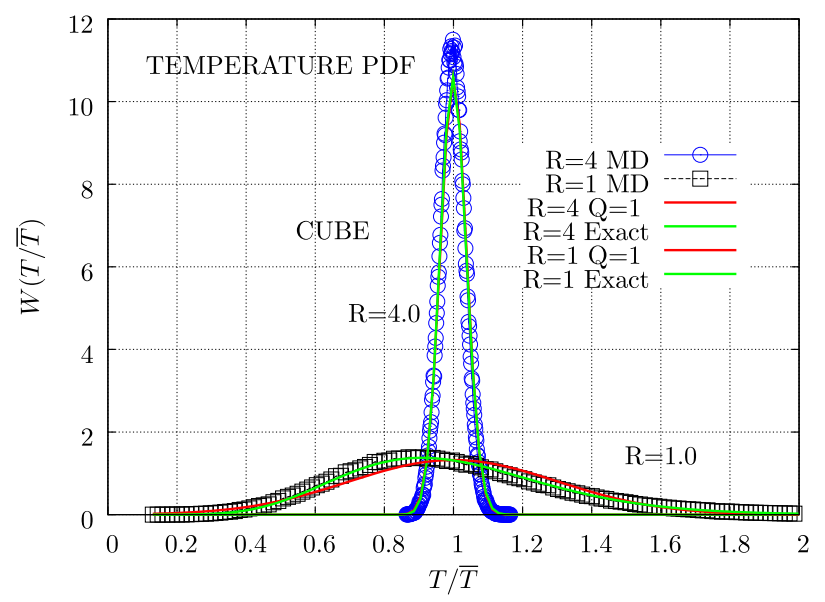

FIG. 9. The normalized local temperature probability distribution function, $W(T / \bar{T})$, using the same system parameters as for Fig. 6. Data for $R=1$ and $R=4$ are given on the figure. The symbols are for NVE MD data. The two red curves are the Gaussian PDF forms from Eq. (15) with a variance taken from Eq. (17), using thermal softness parameter, $Q_{T}=1$ (ideal gas). The green lines are the exact solutions in the thermodynamic limit, from Eq. (21) for $R=1$ and ((22)) for $R=4$ on the figure ("Exact"). For $R=4$ the red and green lines are hardly distinguishable.
The distribution is not quite Gaussian, especially visible for $R=1$. The exact PDF for the mean temperature of the SV taken from the average of the $M$ molecule values is, in fact, if $x=T / \bar{T},{ }^{63}$

$$
W(x)=\frac{1}{x \Gamma(3 M / 2)}\left(3 M x e^{-x} / 2\right)^{3 M / 2},
$$

where $\Gamma(\mathrm{x})$ is the gamma function of $\mathrm{x}$. The temperature PDF, $W(x)$, in Eq. (21) is the ratio of two large numbers for large, $M$. An expansion of the gamma function, ${ }^{68}$ in Eq. (21) gives after some rearrangement,

$$
W(x) \simeq\left(\frac{3 M}{4 \pi}\right)^{1 / 2} \frac{1}{x}\left(x e^{1-x}\right)^{3 M / 2},
$$

which proves more computationally robust in the large $M$ limit, and it is this quantity that is plotted in Fig. 9 for $R=4$. The two red curves are the Gaussian PDF form from Eq. (15) with a variance taken from Eq. (17), using thermal softness parameter, $Q_{T}=1$ (ideal gas). The green lines are the exact solutions in the thermodynamic limit, from Eq. (21) for $R=1$ and (see (22)) for $R=4$ on the figure ("Exact"). For $R=4$ the red and green lines are hardly distinguishable, and the Gaussian approximation is sufficient, whereas for $R=1$ the formula in Eq. (22) gives a slightly better match to the simulation data. Departures from Gaussian statistics become evident for $R$ values smaller than about 1, but there is a gradual deviation (small at first) from Gaussian form as evident in the skew and kurtosis as $R$ decreases from $s / 2$.

The local pressure fluctuation formula for a large system on the molecular scale is $K_{\infty}-K_{0}=\left(V / k_{B} T\right)\left\langle(\Delta P)^{2}\right\rangle .^{64,69}$ This relates the infinite frequency bulk modulus, $K_{\infty}$, and the zero frequency adiabatic bulk modulus, $K_{0},{ }^{70}$ to the volume of the system, $V$, and the mean square difference in the pressure from the mean value, i.e., $\Delta P=P-\bar{P}$. This formula is derived for the whole domain (i.e., where $V$ is the volume of the simulation cell) and the microcanonical ensemble. ${ }^{65,71,72}$ The pressure fluctuation consists of three terms, a purely kinetic (" $k k$ "), a purely configurational ("cc"), and a cross term (" $2 k c$ "), which means that the fluctuations in the total pressure can be separated additively into these components, as the variance of the pressure fluctuations defined in Eq. (18) is, apart from known constants or averages of the simulation, proportional to $K_{\infty}-K_{0}$. For the $\rho=0.9157$ and $T=1$ state point used in the simulations, the $k k$ value is 0.26 , the $2 k c$ term is -4.27 , the $c c$ term is 18.48 from NVE dynamics, and the total $K_{\infty}-K_{0}$ value is 14.48 .

Figure 10 shows the configurational part of the pressure variance, $\left\langle\left(\Delta P_{c}\right)^{2}\right\rangle$, as a function of $\Omega^{-1}$ on a log-log scale for the cubic and spherical subvolumes (there is no statistically significant difference between the two or for the ensemble). Figure 10 and Table I indicate that the variance of the VA pressure is larger than that of the AVIR method for small subvolumes, indicating a greater deviation from Gaussian form in the VA case. Supplementary material Figs. S1 and S2 show representative examples of the AVIR and VA pressure PDFs, indicating that the VA distribution is typically more skewed than the AVIR case for $R \simeq 1$ and smaller. They are both essentially Gaussian for $R \simeq 2$ and larger. Supplementary material Fig. S3 presents the variance, skew, and kurtosis 


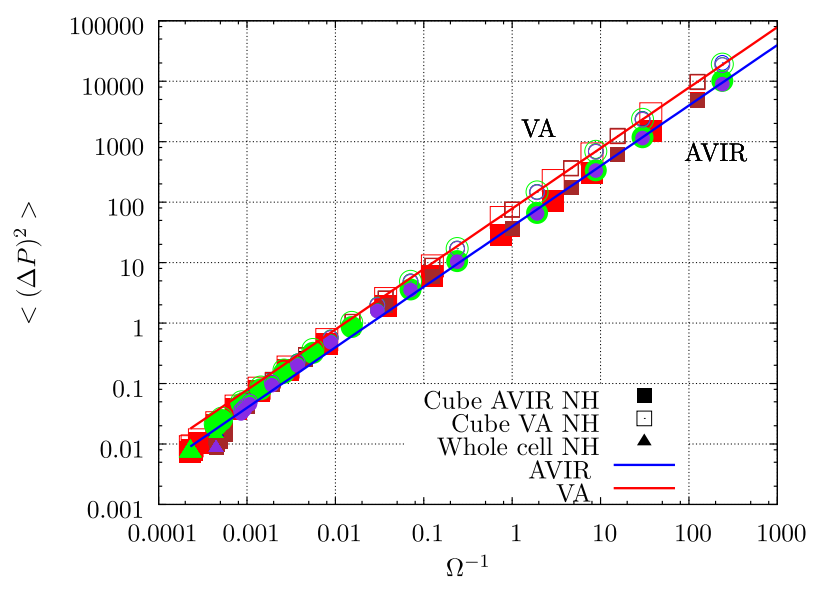

FIG. 10. $\left\langle(\Delta P)^{2}\right\rangle$ as a function of $\Omega^{-1}$ on a $\log -\log$ scale. Data for the cubic and spherical subvolumes are given in the figure. The blue line is $\left\langle(\Delta P)^{2}\right\rangle=A / \Omega$ (where $A$ is an effective " $k_{B} T\left(K_{\infty}-K_{0}\right)$ ") for the AVIR definition of the pressure, and the red line is for the VA definition of the pressure). The linear regression fit is applied to the MD data for $\Omega \leq 1$.0. Note on the $\log -\log$ plot the difference in $A$ is marked by a shift upwards in the two lines with the same slope. The effective moduli values obtained are given in Table I. The state point is $\rho=0.9157$ and $T=1.0$ for $N=2048$ carried out in the microcanonical ensemble (see Ref. 65 p. 92). Key: The square symbols are for cubic subvolumes, and the circles are for the spherical subvolumes. The black, red, and brown symbols are for the NH thermostat, VS thermostat, and NVE ensemble, respectively. The small symbols are for $N=2048$ and the larger ones are for $N=4000$.

statistical of the PDFs as a function of $R$ which supports these conclusions.

Figure 11 shows the variance of the configurational part of shear stress PDF, $\left\langle\left(\Delta P_{x y}\right)^{2}\right\rangle$ as a function of $\Omega^{-1}$. The data for the cubic and spherical subvolumes follow the linear behavior expected from $\left\langle\left(\Delta P_{x y}\right)^{2}\right\rangle=k_{B} T G_{\infty} / \Omega{ }^{64,73}$ The slopes of the lines on the figure in the $\Omega \rightarrow 0$ limit indicate in Table I effective $G_{\infty}$ which are different to the bulk Green-Kubo value of 36.1 for the $c c$ component. Supplementary material Fig. S4 shows some representative shear stress PDFs, and Fig. S5 presents the variance, skew, and kurtosis statistical measures of the shear stress PDFs as a function of $R$, which indicates that generally the shear stress PDFs are more Gaussian than the corresponding pressure PDFs.

TABLE I. The effective static properties obtained by plotting the variance of the property against $\Omega^{-1}$. The state point is $\rho=0.9157$ and $T=1.0$, with $N$ equal to 2048 and 4000. For $\rho$ see Fig. 8, for $P$ see Fig. 10, and for $P_{x y}$ (shear) see Fig. 11. The subscripts $V A$ and $A V I R$ stand for the VA and AVIR definitions of the pressure and shear stress. The static constants in the third column are obtained by least square fitting to the MD data for $\Omega \leq 1.0$. The static constants in the fourth column are for the thermodynamic limit. Note that the equilibrium bulk modulus $K_{T}=\beta_{T}^{-1}$, where $\beta_{T}$ is the isothermal compressibility.

\begin{tabular}{lccr}
\hline \hline Fluctuation property & Static constant & $\Omega \rightarrow 0$ & $\Omega \rightarrow \infty$ \\
\hline$\rho$ & $K_{T}$ & $0.92^{\mathrm{a}}$ & 31.8 \\
$T$ & $C_{v}$ & $1.5^{\mathrm{a}}$ & 1.5 \\
$P_{V A}$ & $K_{\infty}-K_{0}$ & 78.3 & 14.5 \\
$P_{A V I R}$ & $K_{\infty}-K_{0}$ & 39.7 & 14.5 \\
$P_{x y, V A}$ & $G_{\infty}$ & 47.4 & 36.1 \\
$P_{x y, A V I R}$ & $G_{\infty}$ & 19.3 & 36.1 \\
\hline \hline
\end{tabular}

${ }^{\mathrm{a}}$ Ideal gas value.

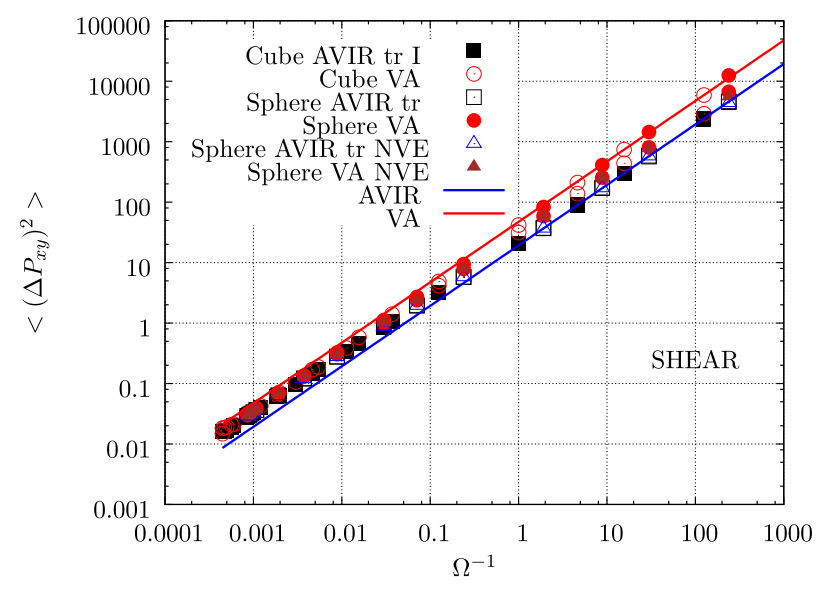

FIG. 11. The variance of the configurational part of the shear stress, $\left\langle\left(\Delta P_{x y}\right)^{2}\right\rangle$ as a function of $\Omega^{-1}$ on a log-log scale. Data for the cubic and spherical subvolumes are given in the figure. The lines are $\left\langle\left(\Delta P_{x y}\right)^{2}\right\rangle=B / \Omega$ (where $B$ is an effective " $k_{B} T G_{\infty, c}$ ") using the VA (upper line) and AVIR (lower line) definitions of the shear stress. The least square fits are applied to the MD data for $\Omega \leq 1.0$, where $G_{\infty, c}=38.7$. Note the log-log scale.

Table I lists the effective thermomechanical properties obtained from the variance of the static property in the $\Omega^{-1} \rightarrow 0$ limit for the density, temperature, pressure, and shear stress, for $\rho=0.9157$ and $T=1.0$. The linear regression treatment of the variances shown in Figs. 8, 10, and 11 were used to determine these values. The table shows that for the $\rho$ and $T$ PDFs the relevant second order thermodynamic property in the small SV limit is the ideal gas value. The $\Omega \rightarrow 0$ apparent elastic moduli obtained from the regression fit are generally higher than their actual values in the thermodynamic limit, meaning that the fluctuations are larger than one would expect based on simple volume scaling of the macroscopic limit formulas.

\section{CONCLUSIONS}

The virial expression for the bulk pressure tensor can be written in part as the sum of an "internal virial" term which should average to zero over a long simulation, but can be slow to converge. The other component ("external virial") is the Method of Planes (MOP) definition applied to the faces of a simulation cell. It is shown that the latter method can be used instead of the full virial formula in certain circumstances where the relative contribution of the internal virial term to the total is small. This reduces the number of interactions that need to be considered, which could be useful for large scale simulations involving multiterm force fields.

The equilibrium probability distribution function (PDF) of the local volume averaged density, temperature, pressure, and shear stress were found to be insensitive to the constant temperature states using velocity scaling or Nosé-Hoover thermostats. Also the PDFs were independent of whether constant temperature or microcanonical (NVE) dynamics were used, in both cases for sampling volumes not too close to the volume of the whole simulation cell. The PDFs are Gaussian in the thermodynamic limit, and it is shown that this behavior continues to a good approximation down 
to subvolumes containing as few as about ten molecules. The variance of the property is inversely proportional to the subvolume, $\Omega$, in each case, using the same expression for the variance of the distribution. The effective moduli tend to be larger than the bulk values if data from the small subvolume limit are included in the fitting process. Below this volume the variance increases more rapidly than $\Omega^{-1}$, reflecting an increasing departure from a Gaussian PDF form. This is especially the case for the VA definition of the pressure. An atomic virial definition of the local pressure tensor results in a distribution which is closer to Gaussian than obtained from volume averaging. Also in the small SV limit the shear stress PDF for all definitions considered is more Gaussian than the pressure at the same subvolume. Some discussion on the relevance of the present results to MD-Continuum coupling is given in the supplementary material.

\section{SUPPLEMENTARY MATERIAL}

See the supplementary material for further details of Eq. (7), the long range correction for MOP, more on local density fluctuations, and examples of the local pressure and shear stress PDFs. Figures showing the $R$-dependence of the variance, skew, and kurtosis of the pressure and shear stress PDFs are also presented.

${ }^{1}$ B. J. Alder and T. E. Wainwright, J. Chem. Phys. 27, 1208 (1957); ibid. 31, 459 (1959).

${ }^{2}$ X. Bian, Z. Li, M. Deng, and G. E. Karniadakis, Phys. Rev. E 92, 053302 (2015).

${ }^{3}$ S. T. O'Connell and P. A. Thompson, Phys. Rev. E 52, R5792 (1995).

${ }^{4}$ X. B. Nie, S. Y. Chen, W. N. E., and M. O. Robbins, J. Fluid Mech. 500, 55 (2004).

${ }^{5}$ N. G. Hadjiconstantinou, A. L. Garcia, M. Z. Bazant, and G. He, J. Comput. Phys. 187, 274 (2003).

${ }^{6}$ R. Delgado-Buscalioni and G. De Fabritis, Phys. Rev. E 76, 036709 (2007).

${ }^{7}$ G. De Fabritis, M. Serrano, R. Delgado-Buscalioni, and P. V. Coveney, Phys. Rev. E 75, 026307 (2007).

${ }^{8}$ A. Amadei, M. E. F. Apol, and H. J. C. Berendsen, J. Chem. Phys. 106, 1893 (1997).

${ }^{9}$ M. P. Allen and D. J. Tildesley, Computer Simulation of Liquids (Clarendon Press, Oxford, 1987), p. 54 Eq. (2.88).

${ }^{10}$ S. Hess and D. J. Evans, Phys. Rev. E 64, 011207 (2001).

${ }^{11}$ S. Hess, M. Kröger, and D. J. Evans, Phys. Rev. E 67, 042201 (2003).

${ }^{12}$ T. Chen, B. Smit, and A. T. Bell, J. Chem. Phys. 131, 246101 (2009).

${ }^{13}$ G.-J. Guo, Y.-G. Zhang, and Y.-J. Zhao, Phys. Rev. E 67, 043101 (2003).

${ }^{14}$ D. J. Evans, E. G. D. Cohen, and G. P. Morriss, Phys. Rev. Lett. 71, 2401 (1993).

${ }^{15}$ D. J. Evans and D. J. Searles, Adv. Phys. 51, 1529 (2002).

${ }^{16}$ J. H. Irving and J. G. Kirkwood, J. Chem. Phys. 18, 817 (1950).

${ }^{17}$ P. Schofield and J. R. Henderson, Proc. R. Soc. A 379, 231 (1982).

${ }^{18}$ J. R. Henderson, J. Chem. Phys. 84, 3385 (1986).

${ }^{19}$ B. Liu and X. Qiu, J. Comput. Theor. Nanosci. 6, 1081 (2009).

${ }^{20}$ A. K. Subramaniyan and C. T. Sun, Int. J. Solids Struct. 45, 4340 (2008).

${ }^{21}$ J. Cormier, J. M. Rickman, and T. J. Delph, J. Appl. Phys. 89, 99 (2001).

${ }^{22}$ J.-P. Hansen and I. R. McDonald, Theory of Simple Liquids, 4th ed. (Academic Press, Amsterdam, 2013), p. 36.
${ }^{23}$ J.-A. Schweitz, J. Phys. A: Math. Gen. 10, 507 (1977).

${ }^{24}$ R. J. Swenson, Am. J. Phys. 51, 940 (1983).

${ }^{25}$ M. J. Louwerse and E. J. Baerends, Chem. Phys. Lett. 421, 138 (2006).

${ }^{26}$ J. H. Hristov, R. Paul, and S. J. Paddison, J. Chem. Phys. 131, 164103 (2009).

${ }^{27}$ E. N. Parker, Phys. Rev. 96, 1686 (1954).

${ }^{28}$ B. Widom, Statistical Mechanics: A Concise Introduction for Chemists (Cambridge UP, Cambridge, 2002), pp. 118-124.

${ }^{29}$ B. D. Todd, P. J. Daivis, and D. J. Evans, Phys. Rev. E 51, 1627 (1995).

${ }^{30}$ D. M. Heyes, E. R. Smith, D. Dini, and T. A. Zaki, J. Chem. Phys. 135, 024512 (2011).

${ }^{31}$ H. Heinz, W. Paul, and K. Binder, Phys. Rev. E 72, 066704 (2005).

${ }^{32}$ H. Heinz, Mol. Simul. 33, 15 (2007).

${ }^{33}$ D. M. Heyes, The Liquid State (John Wiley \& Sons, Chichester, 1997).

${ }^{34}$ D. M. Heyes, E. R. Smith, D. Dini, and T. A. Zaki, J. Chem. Phys. 140, 054506 (2014).

${ }^{35}$ E. R. Smith, D. M. Heyes, D. Dini, and T. A. Zaki, Phys. Rev. E 85, 056705 (2012).

${ }^{36}$ E. M. Blokhuis and D. Bedeaux, J. Chem. Phys. 97, 3576 (1992).

${ }^{37}$ S. M. Thompson, K. E. Gubbins, J. P. R. B. Walton, R. A. R. Chantry, and J. S. Rowlinson, J. Chem. Phys. 81, 530 (1984).

${ }^{38}$ A. P. Shreve, J. P. R. B. Walton, and K. E. Gubbins, J. Chem. Phys. 85, 2178 (1986).

${ }^{39}$ G. J. Tjatjopolous and J. A. Mann, Mol. Phys. 60, 1425 (1987).

${ }^{40}$ M. Rao and B. J. Berne, Mol. Phys. 37, 455 (1979).

${ }^{41}$ N. W. A. van Uden, H. Hubel, D. A. Faux, A. C. Tanczos, B. Howlin, and D. J. Dunstan, J. Phys.: Condens. Matter 15, 1577 (2003).

${ }^{42}$ J. Ding and Y. Cheng, Appl. Phys. Lett. 104, 051903 (2014).

${ }^{43}$ K. S. Cheung and S. Yip, J. Appl. Phys. 70, 5688 (1991).

${ }^{44}$ L. V. Woodcock, Chem. Phys. Lett. 10, 257 (1971).

${ }^{45}$ L. V. Woodcock, CCP5 Information Quarterly No. 24, March 1987, p. 297.

${ }^{46}$ S. Nosé, Mol. Phys. 52, 255 (1984).

${ }^{47}$ W. G. Hoover, Phys. Rev. A 31, 1695 (1985).

${ }^{48}$ S. Nosé, Prog. Theor. Phys. Suppl. 103, 1 (1991).

${ }^{49}$ D. M. Heyes and A. C. Brańka, J. Chem. Phys. 143, 234504 (2015).

${ }^{50}$ J. Petravic, J. Chem. Phys. 120, 7041 (2004).

${ }^{51}$ S. Abraham and P. Harrowell, J. Chem. Phys. 137, 014506 (2012).

${ }^{52}$ I. Fuereder and P. Ilg, J. Chem. Phys. 142, 144505 (2015).

${ }^{53} \mathrm{M}$. González-Melchor, P. Orea, J. López-Lemus, F. Bresme, and J. J. Alejandre, J. Chem. Phys. 122, 094503 (2005).

${ }^{54}$ S. R. Williams, J. Chem. Phys. 135, 131102 (2011).

${ }^{55}$ L. R. Pratt and S. H. Haan, J. Chem. Phys. 74, 1864 (1981).

${ }^{56}$ J. D. Weeks, D. Chandler, and H. C. Andersen, J. Chem. Phys. 54, 5237 (1971).

${ }^{57}$ D. A. McQuarrie, Mathematical Methods for Scientists and Engineers (University Science Books, Sausalito, California, 2003), p. 1046.

${ }^{58}$ N. H. March and M. P. Tosi, Introduction to Liquid State Physics (World Scientific, New Jersey, 2002), pp. 59-62.

${ }^{59}$ M. Rovere, D. W. Hermann, and K. Binder, Europhys. Lett. 6, 585 (1988).

${ }^{60}$ I. R. McDonald and K. Singer, Mol. Phys. 23, 29 (1972).

${ }^{61}$ V. V. Nauchitel, Mol. Phys. 42, 1259 (1981).

${ }^{62}$ D. Pollard, J. Chem. Phys. 112, 6554 (2000).

${ }^{63}$ R. J. Dimelow and A. J. Masters, Mol. Simul. 33, 1165 (2007).

${ }^{64}$ J. G. Powles and D. M. Heyes, Mol. Phys. 98, 917 (2000).

${ }^{65}$ R. Zwanzig, Annu. Rev. Phys. Chem. 16, 67 (1965).

${ }^{66}$ G. Rickayzen, J. G. Powles, and D. M. Heyes, J. Chem. Phys. 118, 11048 (2003).

${ }^{67}$ J. Kolafa and I. Nezbeda, Fluid Phase Equilib. 100, 1-34 (1994).

${ }^{68}$ M. Abramowitz and I. A. Stegun, Handbook of Mathematical Functions (Dover Publications, New York, 1972), p. 257 Eq. 6.1.37.

${ }^{69}$ D. M. Heyes, G. Rickayzen, and A. C. Brańka, Mol. Phys. 102, 2057 (2004).

${ }^{70}$ D. Levesque, L. Verlet, and J. Kürijarvi, Phys. Rev. A 7, 1690 (1973).

${ }^{71}$ G. A. Fernández, J. Vrabec, and H. Hasse, Fluid Phase Equilib. 221, 157 (2004).

${ }^{72}$ J. A. McLennan, Prog. Theor. Phys. 30, 408 (1963).

${ }^{73}$ H. Stassen and W. A. Steele, J. Chem. Phys. 102, 8533 (1995). 